\section{DuD - Datenschutz und Datensicherheit}

Recht und Sicherheit in Informationsverarbeitung und Kommunikation 31. Jahrgang, Heft 7, Juli 2007

Friedr. Vieweg \& Sohn Verlag / GWV Fachverlage GmbH Abraham-Lincoln-Straße 46, 65189 Wiesbaden

\section{Geschäftsführer: Andreas Kösters Albrecht F. Schirmacher Dr. Ralf Birkelbach}

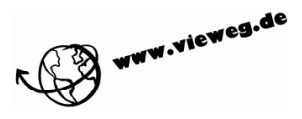

Gesamtleitung Produktion: Ingo Eichel Gesamtleitung Anzeigen: Thomas Werner

Herausgeber:

Prof. Dr. H. Reimer

Chamissostrasse 11, 99096 Erfurt

Telefon: (0361) 3460531, Telefax: (0361) 3453957

E-Mail: helmut.reimer@dud.de

Dr. J. Bizer

Nelkenweg 16, 24147 Kiel

Telefon: (0431) 98812-86, Telefax: (0431) 98812-23

E-Mail: johann.bizer@dud.de

Dipl.-Inform. D. Fox

Ettlinger Strasse 12-14, 76137 Karlsruhe

Telefon: (0721) 255171-203, Telefax: (0721) 255171-100

E-Mail: dirk.fox@dud.de

Seniorherausgeber:

Dr. K. Rihaczek, Bad Homburg

Beirat:

Dr. B. Beier, Lohmar, Konzerndatenschutzbeauftragter GKN

Prof. Dr. H. Burkert, St. Augustin, GMD und Universität St. Gallen

Prof. Dr. A. Büllesbach, Stuttgart, Konzernbeauftragter für den Datenschutz der DaimlerChrysler AG

Prof. Dr. R.W. Gerling, München, Datenschutzbeauftragter der Max-Planck-Gesellschaft

LL.M Gabriela Krader, Bonn, Konzern-Datenschutzbeauftragte Deutsche Post World Net

Prof. Dr. R. Grimm, Koblenz, Institut für Wirtschafts- und Verwaltungsinformatik der Universität Koblenz-Landau

Prof. Dr. P. Horster, Klagenfurt, Institut für Systemsicherheit an der Universität Klagenfurt

Th. Königshofen, ZB Konzernsicherheit, Deutsche Telekom AG

W. Niehoff, München, HypoVereinsbank

Prof. Dr. Sachar Paulus, Walldorf, Security Strategist, SAP AG

Prof. Dr. A. Pfitzmann, Dresden, Fakultät Informatik der Technischen Universität

Prof. Dr. A. Roßnagel, Projektgruppe verfassungsverträgliche Technikgestaltung (provet), Universität Kassel

P. Schaar, Bundesbeauftragter für den Datenschutz

Prof. Dr. R. Schweizer, Professor an der Hochschule St. Gallen

Prof. Dr. Marie Theres Tinnefeld, München, Juristin, Publizistin

Dr. M. Waidner, Zürich, IBM Forschungslaboratorium

Reinhard Vetter, München, Bayrischer Landesbeauftragter für den Datenschutz a.D.

K.-D. Wolfenstetter, Darmstadt, Abteilungsleiter Software-Techniken und Systemsicherheit, TZ der Deutschen Telekom

\section{Technische Redaktion:}

Oliver Reimer

Herderstraße 4, 98693 Ilmenau

Telefon: (03677) 208981, Telefax: (03677) 208982

E-Mail: oliver.reimer@dud.de
Abonnentenverwaltung:

Ursula Müller, Telefon: (05241) 801965, Telefax: (05241) 809620

E-Mail: ursula.mueller@bertelsmann.de

Marketing:

Eva Brechtel-Wahl

Telefon: (0611) 7878-379, Telefax: (0611) 7878-439

E-Mail: eva.brechtel-wahl@gwv-fachverlage.de

Anzeigen:

Anzeigenleitung

Christian Kannenberg, Telefon: (0611) 7878-369, Telefax: (0611) 7878-430

E-Mail: christian.kannenberg@gwv-fachverlage.de

Anzeigendisposition:

Barbara Gerlach, Telefon: (0611) 7878-198, Telefax: (0611) 7878-443

E-Mail: barbara.gerlach@gwv-fachverlage.de

Es gilt die Anzeigenpreisliste von 01.01.2007

Produktion: Frieder Kumm

\section{Bezugsmöglichkeiten:}

Jährlich 12 Hefte. 1-Jahresabonnement (2007) EUR 261,-

(Versandkosten Inland EUR 16,-)

2-Jahresabonnement (2007/2008) EUR 469,-

(Versandkosten Inland EUR 29,-)

Einzelheft EUR 28,-

Alle Bezugspreise und Versandkosten unterliegen der Preisbindung.

Jahresabonnement 2007 zum Vorzugspreis EUR 131,- zuzüglich Versandkosten, gültig für persönliche Mitglieder der AwV (Arbeitsgemeinschaft für wirtschaftliche Verwaltung), des BvD (Berufsverband der Datenschutzbeauftragten Deutschlands e.V.), des BVIT (BundesVerband InformationsTechnologien e.V.), der DVD (Deutschen Vereinigung für Datenschutz e.V.), der DGRI (Deutsche Gesellschaft für Recht und Informatik), des FIfF (Forum Informatiker/Innen für Frieden und Gesellschaftliche Verantwortung e.V.), der GI (Gesellschaft für Informatik), sowie für Studenten. Der Vorzugspreis wird eingeräumt, wenn eine Bestätigung der Mitgliedschaft bzw. eine Studienbescheinigung vorgelegt wird.

Bezug durch den Buchhandel oder den Verlag. Abbestellungen müssen schriftlich spätestens 6 Wochen vor Ende des Bezugszeitraumes erfolgen.

Im laufenden Jahrgang kann jeweils ein Sonderheft erscheinen, das nach Umfang berechnet und den Abonnenten im Erscheinungsjahr mit einem Nachlaß von $25 \%$ des jeweiligen Ladenpreises geliefert wird. Bei Nichtgefallen kann das Sonderheft innerhalb einer Frist von 3 Wochen zurückgegeben werden

(C) Friedr. Vieweg \& Sohn Verlag / GWV Fachverlage GmbH, Wiesbaden 2007

Alle Rechte vorbehalten. Kein Teil dieser Zeitschrift darf ohne schriftliche Genehmigung des Verlages vervielfältigt oder verbreitet werden. Unter dieses Verbot fällt insbesondere die gewerbliche Vervielfältigung per Kopie, die Aufnahme in elektronische Datenbanken und die Vervielfältigung auf CD-ROM und allen anderen elektronischen Datenträgern.

Satz: Oliver Reimer, Ilmenau

Druck und Verarbeitung: Wilhelm \& Adam, Heusenstamm

Gedruckt auf säurefreiem und chlorarm gebleichtem Papier

Printed in Germany

ISSN print 1614-0702

Hinweise für Autoren:

Bitte beachten Sie die ausführlichen Informationen unter www.dud.de. Manuskripte möglichst in maschinenlesbarer Form (WinWord-Datei) an den zuständigen Herausgeber (DuD Report: Herr Reimer, Recht: Herr Bizer, Technik: Herr Fox) senden. Leserbriefe an die Herausgeber sind erwünscht, deren Publikation und eventuelle Kürzungen vorbehalten. 